\section{Michigan Technological

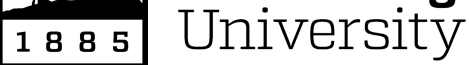

Michigan Technological University Digital Commons@ Michigan Tech

\title{
Self-assembly of 3D nanostructures in electrospun polycaprolactone-polyaniline fibers and their application as scaffolds for tissue engineering
}

Samerender Nagam Hanumantharao

Michigan Technological University

Carolynn Que

Michigan Technological University

Smitha Rao

Michigan Technological University

Follow this and additional works at: https://digitalcommons.mtu.edu/biomedical-fp

Part of the Biomedical Engineering and Bioengineering Commons

\section{Recommended Citation}

Hanumantharao, S. N., Que, C., \& Rao, S. (2019). Self-assembly of 3D nanostructures in electrospun polycaprolactone-polyaniline fibers and their application as scaffolds for tissue engineering. Materialia, 6 . http://dx.doi.org/10.1016/j.mtla.2019.100296

Retrieved from: https://digitalcommons.mtu.edu/biomedical-fp/45

Follow this and additional works at: https://digitalcommons.mtu.edu/biomedical-fp

Part of the Biomedical Engineering and Bioengineering Commons 
Self-Assembly of 3D Nanostructures in Electrospun Polycaprolactone-Polyaniline Fibers and their Application as Scaffolds for Tissue Engineering

Samerender Nagam Hanumantharao, Carolynn Que, Smitha Rao

PII:

DOI:

Article Number:

Reference:

To appear in:

Received date:

Accepted date:
S2589-1529(19)30092-4

https://doi.org/10.1016/j.mtla.2019.100296

100296

MTLA 100296

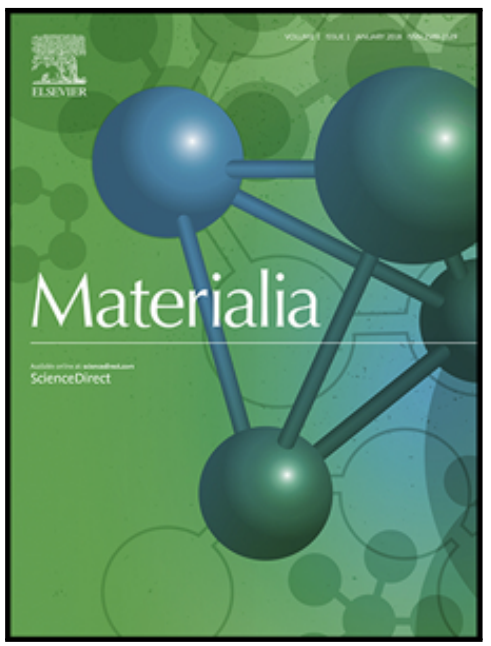

Please cite this article as: Samerender Nagam Hanumantharao, Carolynn Que, Smitha Rao, Self-Assembly of 3D Nanostructures in Electrospun Polycaprolactone-Polyaniline Fibers and their Application as Scaffolds for Tissue Engineering, Materialia (2019), doi: https://doi.org/10.1016/j.mtla.2019.100296

This is a PDF file of an unedited manuscript that has been accepted for publication. As a service to our customers we are providing this early version of the manuscript. The manuscript will undergo copyediting, typesetting, and review of the resulting proof before it is published in its final form. Please note that during the production process errors may be discovered which could affect the content, and all legal disclaimers that apply to the journal pertain. 


\title{
Self-Assembly of 3D Nanostructures in Electrospun Polycaprolactone-Polyaniline Fibers and their Application as Scaffolds for Tissue Engineering
}

\author{
Samerender Nagam Hanumantharao ${ }^{1}$,Carolynn Que ${ }^{1}$, Smitha Rao ${ }^{1}$ \\ ${ }^{I}$ Department of Biomedical Engineering, Michigan Technological University, Houghton, MI \\ 49931, USA \\ *Email: smithar@mtu.edu
}

Keywords: Electrospinning; Nanofibers; Scaffolds; 3D nanostructures

\begin{abstract}
:
The fabrication of synthetic scaffolds that mimic the microenvironment of cells is a crucial challenge in materials science. The honeycomb morphology is one such bio-mimicking structure that possesses unique physical properties and high packing efficiency in a 3dimensional space. Here, we present a novel method for electrospinning polycaprolactonepolyaniline with continuous, self-assembled, uniform, interwoven nanofibers forming patterns without the use of templates or porogens. By using the approach presented here, unique architectures mimicking the natural mechanical anisotropy of extracellular matrix were created by varying the electric field. Adult human dermal fibroblasts (HDFa) cells were successfully cultured on the nanofiber scaffolds without any external growth factors or post-processing of the nanofibers and compared to a commercially available dermal template. Our data indicates that despite identical chemical composition, the physical properties impact cell attachment, alignment and penetration into the scaffold. The mechanical strength of the fibers also plays a role with a distinct preference to fibers with high stiffness and ultimate tensile strength. Thus, by tuning the electric field, fibers with different physical properties and patterns can be fabricated for different applications.
\end{abstract}




\section{Introduction}

The process of self-assembly refers to the efficient and spontaneous bottom-up arrangement of nanostructures with well-defined symmetry and long-range order [1]. Some of the applications of these self-assembled nanostructures are in designing catalysts [2], optics [3], batteries [4], supercapacitors [5], filters with molecular sieves [6], scaffolds for engineering the 3D microenvironment of tissues [7], and drug delivery applications [8]. Self-directed assembly of nanomaterials has been of considerable interest and has gained momentum recently due to advances in fabrication techniques and ability to automate the production. This is critical for the commercial production of functional structures using templating approaches with nanometer scale accuracy. The fabrication of the self-assembled nanostructures can be achieved by mixing colloidal solutions, nanostructured templating, self-organizing medium and wrapping [9]. However, as the complexity of the structure increases, the template and the self-assembled structures will involve integration of multiple processes each with their specific material and chemical requirements.

The formation of different nanostructures including nanodots and nanofibers, can be achieved through electrospinning, an electric-field assisted fabrication technique [10]. The advantages include low cost, quick processing speeds, long-term stability, ease of control and scalability. Nanofibers obtained from electrospinning have been reported for drug delivery [11], tissue scaffold engineering [12], energy storage devices [13], actuators [14], solid state devices [15], smart textiles [16], filters [17], optoelectronics [18], resonators [19], and wound healing [20]. However, the limitation is in efficiently producing nanostructures with high aspect ratio. To address this challenge, modifications such as low temperature electrospinning [21], self- 
assembly [22], replacement of collector with a template of required pattern or liquid bath [2325], post-processing and additive manufacturing have all been reported [26].

The honeycomb topology can be broadly classified based on the shape of the $2 \mathrm{D}$ unit cell type such as square, circular, triangular and hexagonal which is stretched in columns to create 3D structures. The topology of the unit cell is optimized based on the required function. The hexagonal cells help in convective transfer whereas the triangular honeycomb possess superior mechanical properties. This makes the honeycomb structure extremely useful in designing complex nanostructures at the macro- and nanoscale. The packing and tiling of honeycomb structures is also very efficient which allows for high packing densities and interactions between multiple points in a plane [27]. Using electrospinning, the honeycomb structure has been previously reported for energy storage applications [28], tissue scaffold engineering [29], and for use as filters [30]. The pattern offers a wide range of flexibility in design by varying the pore size. Thandavamoorthy et al. demonstrated the production of self-assembled honeycomb structures in membranes using polyurethane [31]. Electrospraying and electrospinning was used by Wittmer to generate 3D honeycomb structures using a patterned collector [32]. Lavielle and colleagues used photolithography to make special patterned collectors composed of conducting and dielectric materials [33]. The fibers were collected in the conducting parts of the collectors and thus provided control over the 3D constructs. Reis and colleagues investigated the selfassembly of the nanofibers to form various 3D structures and the dependence on parameters in forming the structures [34]. The investigations were limited to homogenous polymers and the constituent nanofibers had no alignment. Since the pattern relied on the underlying template on the collector, altering the pores in the structures required changing the collector, thereby limiting dynamic alterations of the structures formed. Nedjari and colleagues fabricated honeycomb 
shaped scaffolds using a patterned template for tissue engineering studies [35]. While the study explored the effect of geometric cues provided by the honeycomb structures, the relation between the variations in the size of the pores to the cell behavior was not reported. Garcia et al. demonstrated the fabrication of honeycomb structures composed of PCL and hydroxyapatite using a patterned collector for bone tissue engineering [36]. A combination of electrospinning and electrospraying was used to obtain the combination of two polymers.

In this paper, a novel technique for self-assembly of nanofibers to form varying patterns of honeycomb structure using electrospinning is presented. This is the first study to report a template free method for the self-assembly of nanofibers to form a pattern with long-range order using polycaprolactone and polyaniline (PCL-PANI). A blend of polymers was used to obtain the required polarity to create the nanopatterns. The size of the pores formed on a standard rotating collector was controlled by changing the electric field alone without any further changes to the electrospinning set-up. The 3D nanostructure thus obtained has the potential for use in drug delivery and energy storage applications with a demonstrated use in tissue engineering.

\section{Experimental Section}

\subsection{Fabrication of Scaffolds}

All the materials were used as procured unless specified. Polycaprolactone (PCL): (MW = 70,000 GPC; Scientific Polymer Products, USA) and Polyaniline (PANI): Emeraldine base $(\mathrm{MW}=50,000 ;$ Aldrich Chemistry, USA) were used for making the nanostructures. The doped form of PANI-PCL solution was prepared by dissolving equal concentrations of $0.35 \%$ wt. emeraldine base and 10-camphorsulfonic acid (CSA) (Aldrich Chemistry; France), and 15\% PCL in chloroform (Sigma Aldrich, USA) and filtering the solution using a syringe filter $(0.22 \mu \mathrm{m})$. The concentration of PANI in the filtered solution was determined post filtration using UV-vis 
spectrophotometer (LAMBDA 35; PerkinElmer, Inc). For electrospinning, the voltage was varied between $18-24 \mathrm{kV}$ in steps of $1 \mathrm{kV}$ to obtain different nanostructures using a rotating cylinder (EM-DIG and EM-RTC; IME Technologies, Netherlands) as collector. The environmental conditions were maintained as a constant. The tip-collector distance was maintained constant at $0.17 \mathrm{~m}$.

\subsection{Characterization of scaffolds}

The nanofibers were coated with a $5 \mathrm{~nm}$ thick coating of $\mathrm{Au} / \mathrm{Pd}$ before visualizing the fibers using a Field emission scanning electron microscope (FESEM; Hitachi S-4700 FE-SEM). The chemical bonds present in the scaffolds were analyzed using an Attenuated Fourier Transform Infrared Spectroscopy (Thermo Scientific ${ }^{\mathrm{TM}}$, Nicolet ${ }^{\mathrm{TM}}$ iS50 FTIR Spectrometer). Dynamic mechanical analyzer (TA Instruments ${ }^{\mathrm{TM}}$, DMA Q800) was used for characterizing the mechanical anisotropy of the scaffolds. The scaffolds were stretched along the direction of alignment of the fibers under isothermal conditions $\left(30^{\circ} \mathrm{C}\right)$. All the characterization experiments were repeated at least thrice to make sure the data was statistically significant.

\subsection{Cell Culture}

Adult Human Dermal Fibroblasts (HDFa) were purchased from America Type Cell Culture (ATCC@ PCS-201-012 ${ }^{\mathrm{TM}}$ ), and were cultured in Dulbecco's modified eagle medium, DMEM, (Life Technologies, USA) supplemented with $10 \%$ fetal bovine serum (Life Technologies, USA) along with 1\% Penicillin Streptomycin (Life Technologies, USA) under standard culture conditions $\left(37^{\circ} \mathrm{C}, 65 \% \mathrm{RH}\right.$ and $\left.5 \% \mathrm{CO}_{2}\right)$. The PCL-PANI scaffolds were cut into squares of $0.5 \mathrm{~cm}^{2}$ area (approximately), sterilized using ethanol, and irradiated in UV. Cells in exponential growing phase were trypsinized, counted using a Bright-Line ${ }^{\mathrm{TM}}$ hemocytometer, 
seeded (2500 cells per scaffold) and cultured in the incubator under standard cell culture conditions.

\subsubsection{Cell characterization}

Cells were visualized via fluorescence microscopy after 2, 3 and 7 days of growth. The cells were stained with DAPI (4',6-diamidino-2-phenylindole) (Life Technologies, USA) and Alexa Fluor ${ }^{\circledR} 488$ Phalloidin (Life Technologies, USA) for nuclei and cytoskeletal F-Actin visualization, respectively according to manufacturer's protocols.

\subsubsection{Cell Viability}

The proliferation studies of fibroblasts on the scaffolds were determined using the CellTiter-Blue ${ }^{\circledR}$ Cell Viability Assay (Promega, Madison, WI). A commercially available wound healing dressing (Hollister Endoform Dermal Template Collagen Dressing) was used as the positive control. The scaffolds including the positive control were cut into squares of side $0.5 \mathrm{~cm}(\mathrm{n}=3)$, placed in sterile 48 -wellplates and 1000 cells per scaffold were seeded. At Days 1 , 3 and 5 culture media was replaced and $20 \%$ volume of CellTiter-Blue ${ }^{\circledR}$ Cell Viability Assay reagent was added and incubated for $4 \mathrm{~h}$ under standard culture conditions $\left(37^{\circ} \mathrm{C}, 65 \% \mathrm{RH}\right.$ and $5 \% \mathrm{CO}_{2}$ ). A $75 \mu \mathrm{L}$ aliquot of the incubated medium was transferred to a 96- well opaque culture plate (Corning Glass Works). Cell viability was determined using a plate reader (Beckman Coulter DTX 880 Multimode Detector) at $560 \mathrm{~nm}$ excitation and $590 \mathrm{~nm}$ emission. The standard error bars were calculated by determining the standard error of mean.

\section{Results and Discussion}

The self-assembly of the honeycomb structures is a multi-step process which begins with the preparation of the polymer blends. The polymer-solvent interactions play a significant role in not only influencing the viscoelasticity and polarity of the solution but also in the morphology, 
topology, crystallinity, electroactivity and mechanical properties of the fibers. Polycaprolactone (PCL), a commonly used viscoelastic polymer was chosen because of its high elasticity, biocompatibility, biodegradability and its solubility in a wide range of solvents. Polyaniline (PANI) is a semiconducting polymer that is known to be conductive in its salt form. The emeraldine salt is obtained by addition of CSA as a dopant. A miscible binary polymer blend was prepared using chloroform. Chloroform was selected as the solvent bécause of its high intrinsic viscosity, low boiling point and electrical conductivity among all the other organic solvents. Concentrations of PANI and PCL in the blend was prepared according to their solubility parameters in chloroform $[37,38]$. The concentration of PANI in the PCL-PANI blend post-filtration was calculated to be $0.4 \mathrm{mg} / \mathrm{mL}$ using UV-visible spectrophotometry (Figure S1). The concentration of PANI dispersed is low because of the solvent used and the presence of a secondary polymer (PCL) in the blend. The PANI is present in its salt form in the PCL-PANI blend which can be interpreted by the presence of peaks at 433-444 nm and 782-815 nm corresponding to the polaron and exciton transition respectively [39]. The range at which a polymer blend can be electrospun is very narrow [40]. One of the important determinants of electrospinning is the formation of a Taylor cone which is dependent on the surface tension of the polymer blend and applied electric field among others. A stable Taylor cone jet is essential for continuous electrospinning and the change in the Taylor cone formed affects the morphology of the nanofibers [41]. In our study reported here, except for the voltage applied to the nozzle, all the parameters used for the electrospinning process to obtain the different honeycomb patterns were kept constant. 


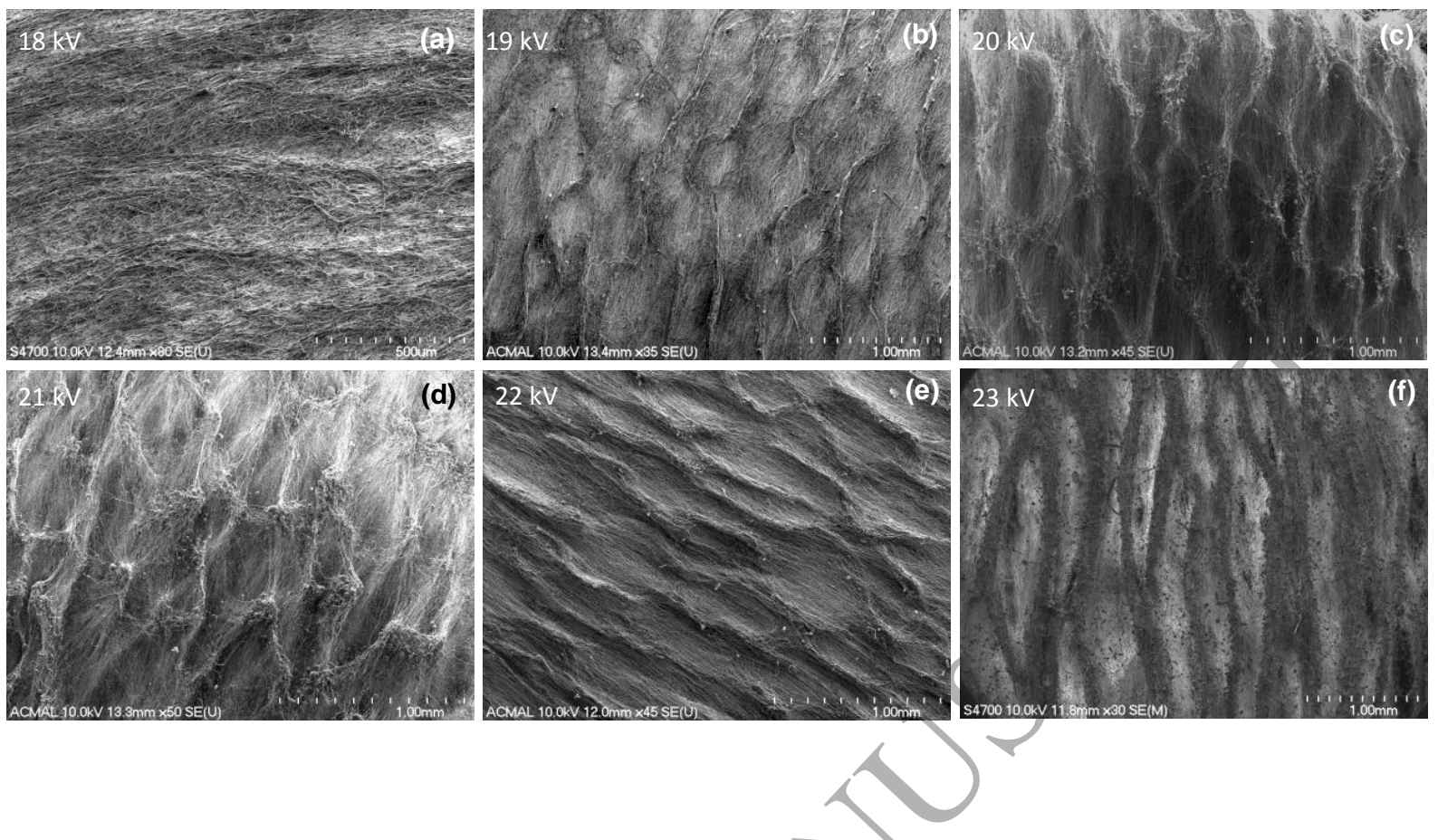

Figure 1. Field emission scanning electron microscopic (FESEM) images of the PCL-PANI scaffolds exhibiting porous morphology and fiber alignment. PCL-PANI-18kV had a wavy architecture without pores (a). The PCL-PANI-19kV the pore formation is visible but is more pronounced in PCL-PANI 20kV and PCL-PANI-21kV (b, c, d). In PCL-PANI-22kV, the structures were elongated and incomplete (e). In PCL-PANI-23kV elongated and closed forming complete, repeatable structures (f).

The low and high magnification FESEM images provide information to relate the effect of electric field during electrospinning to the morphology of the fibers. Low magnification FESEM images of the honeycomb structures formed by electrospinning at different voltages is shown in figure 1(a) through 1(f) and higher magnification FESEM images are presented in supplementary figures S2-S7. At $18 \mathrm{kV}$, the fibers obtained have little to no alignment and there are no interwoven structures or beading, as can be seen from figure 1(a). The higher magnification image (figure S2) shows the formation of clusters of fibers in regions which indicate that a higher electric field (voltage or change in tip-collector distance) and time was 
needed for formation of elongated fibers. The PCL-PANI-19kV, in figure 1(b) have interconnected pores which are small and uneven in arrangement. The honeycomb arrangement cannot be seen uniformly across the entire surface. A higher magnification image (figure S3) of the fibers revealed aligned fibers that are concentrated towards the beads. The underlying structure of the fibers were well aligned and fibrous with little or no beads. This confirms that the formation of beads is not an initial process but occurs at a later point of time during the electrospinning. In PCL-PANI-20kV (figure 1c), the pores are uniaxially aligned and larger than the pores formed at $19 \mathrm{kV}$, with the presence of beading along the perimeters of the pores but not in the other regions. However, a higher magnification image (figure S4) of the scaffold's underlying structure showed beading and a wavy alignment with overlapping layers of fibers forming a tightly packed structure. In PCL-PANI-21kV (figure 1d) deeper pores compared to PCL-PANI-20kV can be observed. A high magnification image (figure S5) of the structure showed the presence of beads and the alignment of the underlying architecture with the fibers concentrated more towards the area containing beads and thus creating pores. The honeycomb pattern in the PCL-PANI-22kV (figure 1e) is stretched out and incomplete and the underlying fibers are aligned with little or no beading. The higher magnification image (figure S6) showed the underlying strueture is completely devoid of beads and droplets. The PCL-PANI-23kV (figure 1f) sample is continuous and has long-range order throughout in a symmetrical manner. The underlying alignment of the fibers is limited to a first few layers of the fibers formed during electrospinning. A high magnification (figure S7) of the regions between the walls of the honeycomb structure and the base layer showed a large concentration of droplets on the walls of the honeycomb structure. The beads act as points of attraction for the fibers to form and this creates a dense network. On a larger scale (millimeter range) this leads to the formation of a 
pattern, a honeycomb structure in this case. Varying the electric field intensity altered the formation, the size of pores, and the nature of the fiber between non-uniform, wavy and straight, since all the other parameters were constant. A high-contrast FESEM image (figure S9) of the fibers helps in highlighting the regions of fiber formation and the size of the pores.

The fibers were collected at a high uptake velocity which decreases the distance between two individual large diameter fibers and at the same time increases alignment rate of the smaller diameter fibers [42]. The underlying structure in each case is a layer of fibers or beads which have a directionality along the rotation of the collector. The time point at which the beads start collecting on the surface of the fibers over the duration of electrospinning is dependent on the electric field. This process fails to start in the case of the fibers electrospun at $18 \mathrm{kV}$ and happens almost immediately in the fibers electrospun at $24 \mathrm{kV}$ (figure S7). The formation of the beads on the fibers causes a change in the distribution of the electrostatic charges. The beads behave in a manner similar to point charges, attracting the fibers towards them in a manner similar to the one reported by Sun et al.[43]. The beads formed have repeated patterns because of the Rayleigh instability and splaying on a rotating collector, finally forming the interconnected honeycomb morphology. The change in the dimensions of the honeycomb structure is in accordance with finding by Reis et al.[34]. Briefly, the experimental verification for the in situ polarization and the factors responsible for electrodynamic tailoring was studied. It was found that the initial regions of high fiber density act as the motifs for the self-assembled process. Attraction to the motifs and repulsion from the other areas favors the growth of the nanostructures. The polarity of the fibers leads to the formation of wavy fibers. The wavy fibers are absent at an increased electric field leading to formation of straight fibers. Increasing the electrical field also increases the surface charge density during electrospinning affecting the shape of the Taylor cone [44]. 
The formation of the Taylor cone was discontinuous at electric field intensities lower than 1.05 $\mathrm{kV} / \mathrm{cm}$. This was due to surface tension and viscosity of the droplet at the needle. The size of the pores of the honeycomb increases gradually with increase in electric field. The Taylor cone then recedes into the needle around an electric field of $1.28 \mathrm{kV} / \mathrm{cm}$. Beyond $1.33 \mathrm{kV} / \mathrm{cm}$, highly beaded structures with very few fibers were formed.
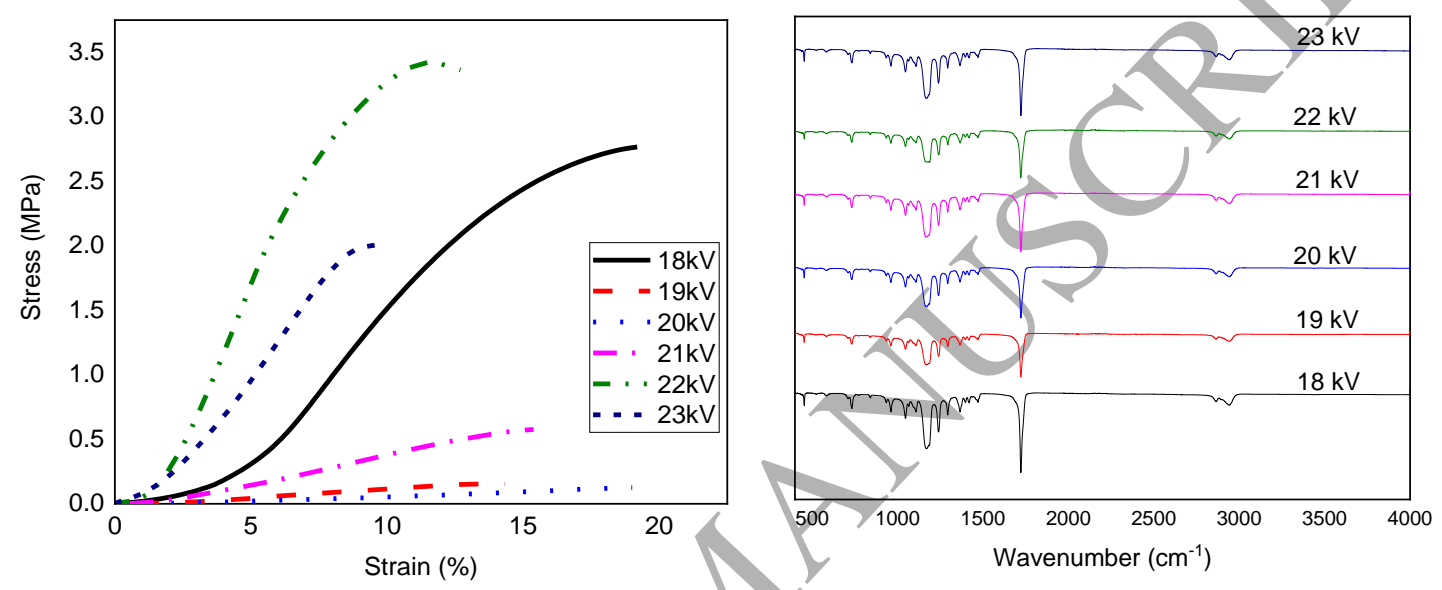

Figure 2A. Mechanical analysis of the different scaffolds illustrated by the stress-strain profiles. PCL-PANI-22kV had the highest ultimate tensile strength while PCL-PANI-20kV had the lowest tensile strength. Figure 2B. Surface analysis of the different scaffolds illustrated by the ATR-FTIR spectra. All the scaffolds had the same functional groups and there was no considerable change in area of the peaks of interest indicating similar surface chemistries between all the scaffolds.

The surface chemistry of the nanofibrous scaffolds were analyzed using ATR-FTIR. A $\%$ Transmittance versus wavenumber plot was generated and signature peaks were identified as shown in data from figure $2 \mathrm{~A}$. The characteristic peaks of $\mathrm{PCL}$, such as the $\mathrm{C}=\mathrm{C}, \mathrm{C}=\mathrm{O}$ are present in all the spectra. The strong intensity peaks at $1722 \mathrm{~cm}^{-1}(\mathrm{C}=\mathrm{O}$ stretching $)$ and $1167(\mathrm{C}$ O stretching), and medium intensity peaks at $2944 \mathrm{~cm}^{-1}$ (asymmetric $\mathrm{CH}_{2}$ stretching) and 2866 
$\mathrm{cm}^{-1}$ (symmetric $\mathrm{CH}_{2}$ stretching) are unique to PCL. The strong intensity peak at $1293 \mathrm{~cm}^{-1}$ is specific to the C-O and C-C stretching in the crystalline phase of PCL. The weak intensity peak at $582 \mathrm{~cm}^{-1}$ is specific to the $\mathrm{C}=\mathrm{O}$ wag in PCL. The characteristic peaks of PCL at $1231 \mathrm{~cm}^{-1}$ (asymmetric C-O-C stretching) and $1176 \mathrm{~cm}^{-1}$ (C-O stretching) confirm the presence of PCL in the PCL-PANI sample. PANI has characteristic peaks in the 3000-3500 region, which is specific to the amine group. The N-H stretching at $3442 \mathrm{~cm}^{-1}$ is specific to PANI. The percentage transmittance of the peaks remained almost similar without significant changes across the scaffolds fabricated at different voltages as inferred from figures S10 and S11.

The mechanical properties of the scaffolds were inferred from the stress-strain plot in figure $2 \mathrm{~B}$ and the analysis from the plot is tabulated in table 1 . The mechanical properties of the scaffold are an important characteristic for cell proliferation, differentiation and migration. The variability in mechanical properties of the scaffolds can also be related with their structures as seen using FESEM. Youngs modulus of the fibers, Ultimate tensile strength and elongation at failure for the scaffolds were extrapolated from the stress-strain relationship of the scaffold. The scaffolds which had a higher percentage of beads have poor mechanical strength indicated by their ultimate tensile strength. The PCL-PANI-19kV, 20kV and $21 \mathrm{kV}$ exhibited poor tensile strength and breakage at low strains. The PCL-PANI- $18 \mathrm{kV}, 22 \mathrm{kV}$ and $23 \mathrm{kV}$ exhibited high stiffness and ultimate tensile strength, which is ideal for culturing fibroblasts $[45,46]$. Thus, the voltage used for fabricating the honeycomb structures enabled modification of the mechanical properties of the scaffold based on the required application [47].

Table 1. Mechanical Analysis of the different samples calculated from the stress-strain profiles.

\begin{tabular}{lcccccc}
\multirow{2}{*}{ Sample } & PCL- & PCL- & PCL- & PCL- & PCL- & PCL- \\
& PANI-18kV & PANI-19kV & PANI-20kV & PANI-21kV & PANI-22kV & PANI-23kV
\end{tabular}




\begin{tabular}{ccccccc}
\hline $\begin{array}{c}\text { Youngs } \\
\text { Modulus }\end{array}$ & $0.178 \pm 0.08$ & $0.009 \pm 0.003$ & $0.009 \pm 0.004$ & $0.051 \pm 0.01$ & $0.42 \pm 0.05$ & $0.271 \pm 0.01$ \\
$(\mathbf{M P a})$ & & & & & & \\
\end{tabular}

\begin{tabular}{ccccccc}
\hline $\begin{array}{c}\text { Ultimate } \\
\text { Tensile }\end{array}$ & & & & & & \\
Strength & $2.089 \pm 0.71$ & $0.179 \pm 0.04$ & $0.148 \pm 0.02$ & $0.506 \pm 0.07$ & $3.68 \pm 0.18$ & $1.748 \pm 0.22$ \\
$($ MPa $)$ & & & & & & \\
\end{tabular}

\begin{tabular}{ccccccc}
\hline Elongation & & & & & & \\
at Fracture & $20.848 \pm 3.98$ & $33.873 \pm$ & $38.337 \pm 20.0$ & $23.030 \pm 14.9$ & $13.47 \pm$ & \\
$(\%)$ & & 17.68 & 2 & 0 & 0.73 & \\
& & & & & &
\end{tabular}

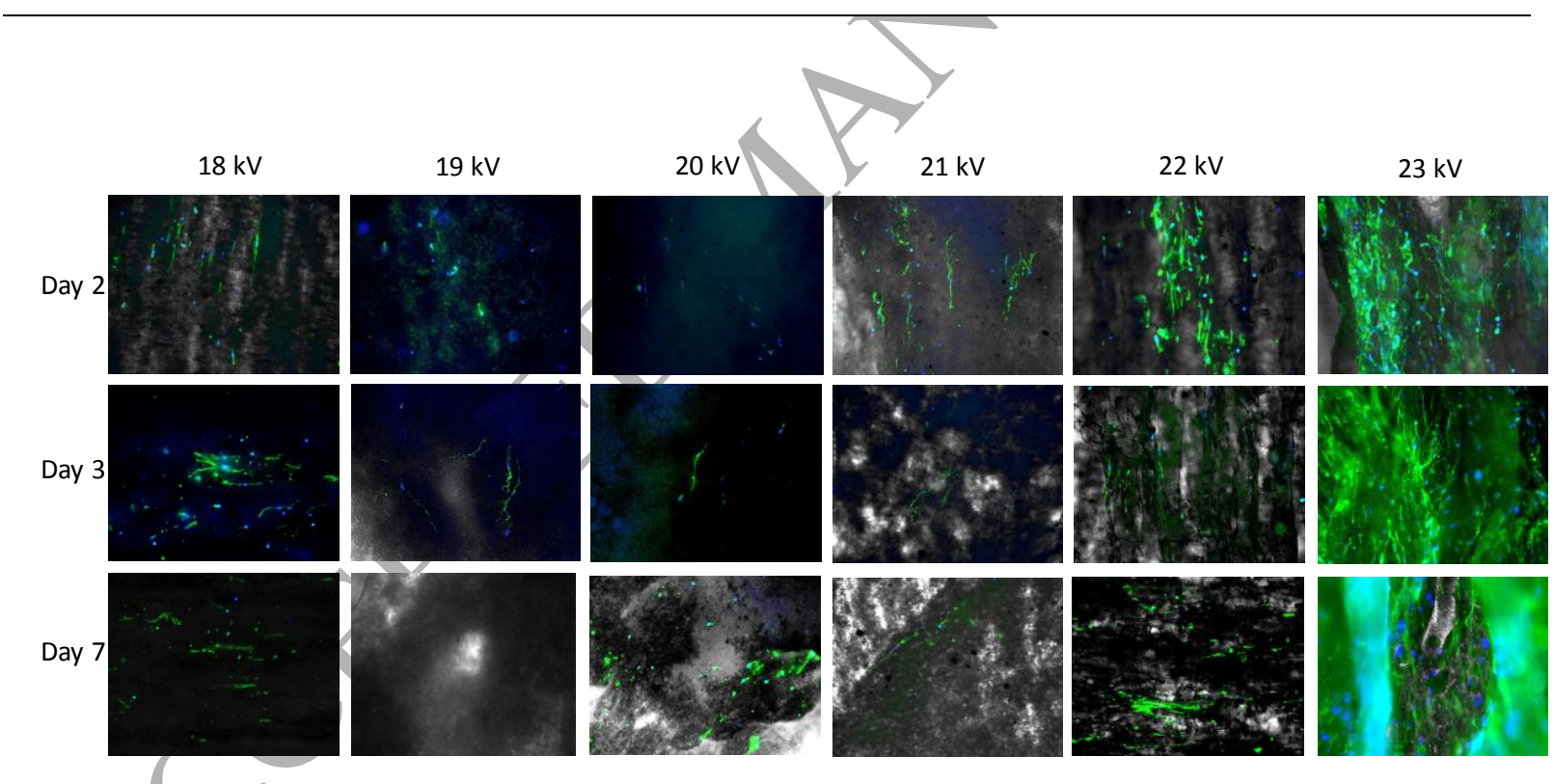

Figure 3. Fluorescent and brightfield overlay microscope images of adult dermal human fibroblast (HDFa) cells on the different scaffolds on days 2, 3 and 7. There appears to be a greater degree cell attachment and alignment on PCL-PANI-23kV for all days. The nuclei were 
stained with DAPI (blue) and F-actin filaments were stained with Alexa Fluor ${ }^{\circledR}$ Phalloidin (green). Images were captured at 10X magnification.

In order to demonstrate feasibility of using PCL-PANI scaffolds in wound healing applications, we carried out cell studies using Adult Human dermal Fibroblast cells (HDFa). The cells act as a good model for in vitro wound healing studies [48]. The scaffolds after cell seeding were fixed and stained with DAPI and Alexa Fluor® 488 Phalloidin to visualize the nucleus and the cytoskeleton (F-actin), respectively as shown in figure 3. The cells were seeded on the sterilized scaffolds without further post-processing or addition of factors to enhance adhesion. Based on the localization of the cells observed after 2 days, they appear to accumulate more towards the edges of the honeycomb structure. The number of cells is more in PCL-PANI-23kV compared to the other scaffolds. From the images, on day 3 , the cells aligned along the direction of alignment of the fibers. By day 7, the cells proliferated along the depth of the scaffold and spread uniformly across the entire scaffold. The cells in PCL-PANI-23kV had the highest cell infiltration while PCL-PANI-19kV had the lowest cell number. The direction of cell alignment was inferred from the brightfield overlay on the fluorescent images outlining the F-actin filaments. The out-of-focus regions in the images are from the multiple layers of the electrospun fiber forming the scaffold. The stiffness of the scaffold helps the cells adhere and grow on it.
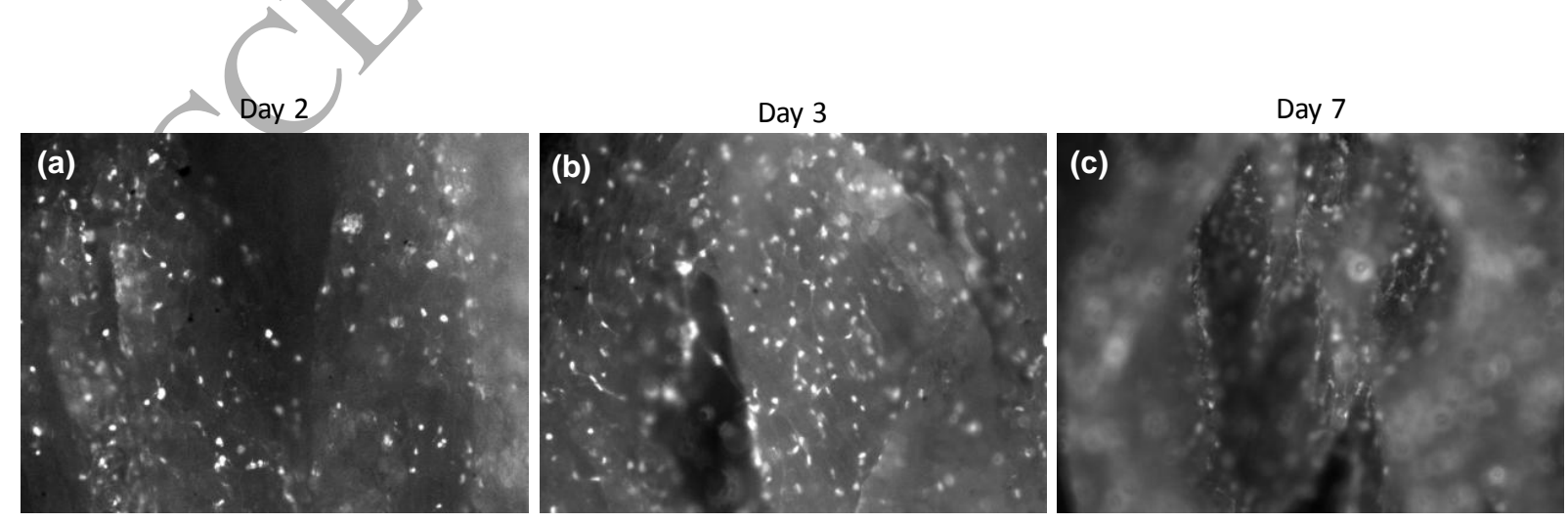
Figure 4. Grayscale images of the fluorescent signals obtained from cell nuclei stained with DAPI (white) demonstrating the localization and infiltration of the dermal fibroblasts on the PCL-PANI-23kV honeycomb structures. The cells remained on the surface on day 2 (a), show proliferation on the surface with some cell infiltration on day 3 (b) with complete infiltration on day 7 (c). All the images were taken at a magnification of $10 \mathrm{X}$.

The shape of the nucleus at different points of the cell seeding for PCL-PANI-23kV (figure 4) shows the synergy of cell, scaffold and topology of the scaffold. The structural characteristics of the scaffold enabled penetration of cells into the lower layers of the scaffold. The cells on day 2 were spread on the upper region of the scaffold. The cell number increased significantly as seen from the number of cells on day 3 with some level of cellular infiltration. The cells on day 7 had completely infiltrated towards the bottom layer of the scaffold. This sort of cell-scaffold interaction helps in increased cell numbers during tissue engineering studies which is essential in applications such as wound healing patches and organ engineering. The PCL in the fibers helps in increasing the elasticity and increase the rupture point of the scaffold. 


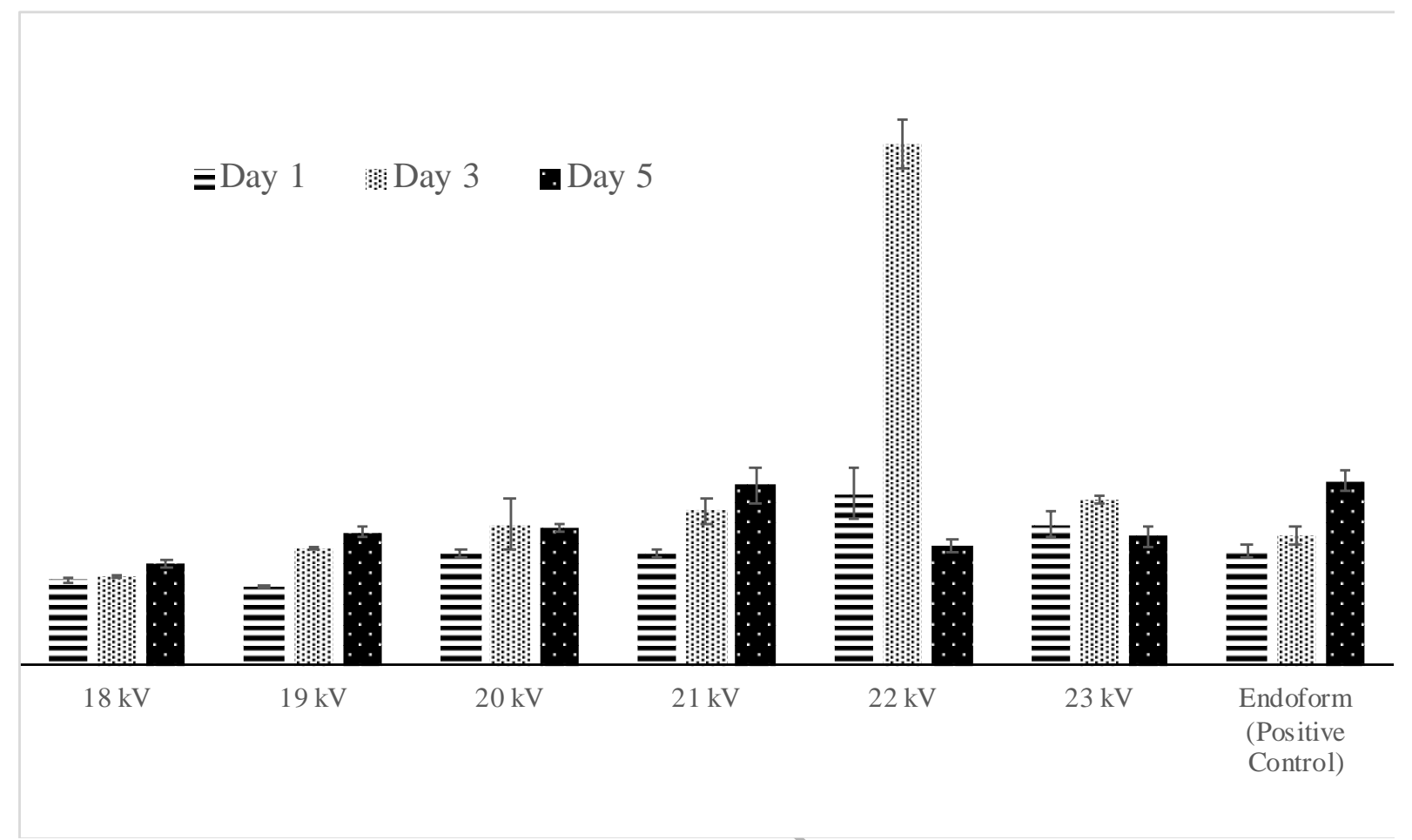

Figure 5. Quantification of cell viability and proliferation on all the scaffolds and controls on Days 1, 3 and 5. Among all the scaffolds, PCL-PANI-22kV and PCL-PANI-23kV had the highest cell viability. While no significant difference was found between PCL-PANI-22kV and $23 \mathrm{kV}$ as compared to the commercially ayailable scaffold, and PCL-PANI- $21 \mathrm{kV}$ nearly identical to the commercial scaffold, the data supports use of synthetic scaffolds for tissue culture applications.

The CellTiter-Blue cell viability assay (figure 5) revealed that the fibroblasts proliferated differently on each scaffold. The cells proliferated more freely on PCL-PANI 20kV through PCL-PANI-23kV. The initial cell proliferation was highest on PCL-PANI-22kV followed by PCL-PANI-23kV. On day 3, the rate of cell proliferation doubled than the rate on day 1 on PCLPANI-22kV. However, on day 5, the cell proliferation decreased for PCL-PANI-22kV and PCLPANI-23kV. This can be attributed to the limited nutrient supply available to the cells since there was no change of media during the study as well as lack of area to further proliferate on the 
scaffolds. This is particularly true in PCL-PANI-20kV through $23 \mathrm{kV}$ as they all have higher cell viability on Day 1 compared to other scaffolds. The cell proliferation was limited on all the three days on PCL-PANI-18kV and PCL-PANI-19kV. The cell proliferation behavior on PCL-PANI$21 \mathrm{kV}$ was similar to the positive control. The accelerated cell proliferation on PCL-PANI-22kV, as indicated by the cell proliferation assay, may not be ideal for wound healing applications but may be useful for rapid regeneration. This aspect requires additional detailed studies to further evaluate the outcomes. The PCL-PANI-23kV and PCL-PANI-22kV have favorable properties for designing an ideal scaffold for wound healing applications. However, PCL-PANI-23kV provides a stable microenvironment for the cells to infiltrate and migrate along the scaffold. The cell proliferation data agrees well with the cell seeding images obtained after immunofluorescent staining.

The morphology of the scaffold plays a significant role in the alignment, infiltration and proliferation of the cells. The cells on PCL-PANI $23 \mathrm{kV}$ have elliptical nucleus and elongated morphologies with the cells distributed throughout the scaffold. The stiffness of the scaffold makes it mechanically compliant for the cells to adhere and proliferate. Furthermore, since the topology and mechanical characteristics are unique to each scaffold, the current method of selfassembly could potentially be used to develop scaffolds to closely match the unique properties of different tissues.

\section{Conclusions}

Here we have successfully demonstrated self-alignment and alteration of the pore size and alignment of electrospun PCL-PANI honeycomb structures without the use of a template. The electric field was controlled to alter the distribution, size and structure of the pattern on the fiber scaffolds. The fibers were characterized for their mechanical strength and surface chemistry, and 
cellular compatibility was demonstrated using adult human fibroblast cells (HDFa). PCL-PANI-

$23 \mathrm{kV}$ had sustainable cellular growth and infiltration among all the other scaffolds.

\section{Acknowledgements}

The authors acknowledge the financial support provided by the Department of Biomedical Engineering at Michigan Technological University and the T3N award from the Michigan Economic Development Corporation. The work was partially supported by financial contribution from Ken Gunther, an alumnus of Michigan Tech. The authors would also like to acknowledge help for characterization studies from Owen Mills at Applied Chemical and Morphological Analysis Laboratory (ACMAL) at Michigan Technological University, Kathryn Perrine, Department of Chemistry for their help with characterization and Infrared Spectroscopy and Chito Kendrick, Managing Director, Microfabrication Facility, Michigan Technological University.

\section{Declaration of Interest}

None

\section{Data Availability}

The processed data required to reproduce these findings have been shared (doi:10.17632/978kbhm3yw.1). The raw data obtained cannot be shared at this time as the data also forms part of an ongoing study.

\section{References}

[1] G.A. Ozin, K. Hou, B.V. Lotsch, L. Cademartiri, D.P. Puzzo, F. Scotognella, A. Ghadimi, J. Thomson, Nanofabrication by self-assembly, Materials Today 12(5) (2009) 12-23.

[2] H.J. Wang, S.L. Yin, Y.H. Li, H.J. Yu, C.J. Li, K. Deng, Y. Xu, X.N. Li, H.R. Xue, L. Wang, One-step fabrication of tri-metallic PdCuAu nanothorn assemblies as an efficient catalyst for oxygen reduction reaction, Journal of Materials Chemistry A 6(8) (2018) 3642-3648.

[3] Y.H. Chen, Z.W. Wang, Y.J. He, Y.J. Yoon, J.H. Jung, G.Z. Zhang, Z.Q. Lin, Light-enabled reversible selfassembly and tunable optical properties of stable hairy nanoparticles, Proc. Natl. Acad. Sci. U. S. A. 115(7) (2018) E1391-E1400. 
[4] H.W. Long, W. Zeng, H. Wang, M.M. Qian, Y.H. Liang, Z.C. Wang, Self-Assembled Biomolecular 1D Nanostructures for Aqueous Sodium-lon Battery, Advanced Science 5(3) (2018).

[5] L. Yu, H.B. Wu, X.W.D. Lou, Self-Templated Formation of Hollow Structures for Electrochemical Energy Applications, Accounts of Chemical Research 50(2) (2017) 293-301.

[6] P. Bengani, Y. Kou, A. Asatekin, Zwitterionic copolymer self-assembly for fouling resistant, high flux membranes with size-based small molecule selectivity, Journal of Membrane Science 493 (2015) 755765.

[7] S. Guven, P. Chen, F. Inci, S. Tasoglu, B. Erkmen, U. Demirci, Multiscale assembly for tissue engineering and regenerative medicine, Trends in biotechnology 33(5) (2015) 269-279.

[8] N. Habibi, N. Kamaly, A. Memic, H. Shafiee, Self-assembled peptide-based nanostructures: smart nanomaterials toward targeted drug delivery, Nano Today 11(1) (2016) 41-60.

[9] S. Mann, Self-assembly and transformation of hybrid nano-objects and nanostructures under equilibrium and non-equilibrium conditions, Nature materials 8(10) (2009) 781

[10] Y.P. Ding, W. Li, F. Zhang, Z.H. Liu, N.Z. Ezazi, D.F. Liu, H.A. Santos, Electrospun Fibrous Architectures for Drug Delivery, Tissue Engineering and Cancer Therapy, Advanced Functional Materials 29(2) (2019). [11] Z. Aytac, Z.I. Yildiz, F. Kayaci-Senirmak, T. Tekinay, T. Uyar, Electrospinning of cyclodextrin/linaloolinclusion complex nanofibers: Fast-dissolving nanofibrous web with prolonged release and antibacterial activity, Food Chemistry 231 (2017) 192-201.

[12] Q.Q. Yao, J.G.L. Cosme, T. Xu, J.M. Miszuk, P.H.S. Picciani, H. Fong, H.L. Sun, Three dimensional electrospun PCL/PLA blend nanofibrous scaffolds with significantly improved stem cells osteogenic differentiation and cranial bone formation, Biomaterials 115 (2017) 115-127.

[13] Q. Liu, J.H. Zhu, L.W. Zhang, Y.J. Qiu, Recent advances in energy materials by electrospinning, Renewable \& Sustainable Energy Reviews 81 (2018) 1825-1858.

[14] C.H. Hong, S.J. Ki, J.H. Jeon, H.L. Che, I.K. Park, C.D. Kee, I.K. Oh, Electroactive bio-composite actuators based on cellulose acetate nanofibers with specially chopped polyaniline nanoparticles through electrospinning, Composites Science and Technology 87 (2013) 135-141.

[15] Y. Zhai, X. Bai, H.N. Cui, J.Y. Zhu, W. Liu, T.X. Zhang, B. Dong, G.C. Pan, L. Xu, S. Zhang, H.W. Song, Carbon dot/polyvinylpyrrolidone hybrid nanofibers with efficient solid-state photoluminescence constructed using an electrospinning technique, Nanotechnology 29(2) (2018).

[16] W.Y. Shao, M. Tebyetekerwa, I. Marriam, W.L. Li, Y.Z. Wu, S.J. Peng, S. Ramakrishna, S.Y. Yang, M.F. Zhu, Polyster@MXene nanofibers-based yarn electrodes, Journal of Power Sources 396 (2018) 683-690.

[17] Y. Liao, C.H. Loh, M. Tian, R. Wang, A.G. Fane, Progress in electrospun polymeric nanofibrous membranes for water treatment: Fabrication, modification and applications, Progress in Polymer Science 77 (2018) 69-94.

[18] F. Mehrpouya, J. Foroughi, S. Naficy, J.M. Razal, M. Naebe, Nanostructured Electrospun Hybrid Graphene/Polyacrylonitrile Yarns, Nanomaterials 7(10) (2017).

[19] D. Chen, L. Yang, W.H. Yu, M.Z. Wu, W. Wang, H.F. Wang, Micro-Electromechanical Acoustic Resonator Coated with Polyethyleneimine Nanofibers for the Detection of Formaldehyde Vapor, Micromachines 9 (2) (2018).

[20] R. Li, Z.Q. Cheng, R.C. Wen, X.D. Zhao, X.B. Yu, L. Sun, Y.Y. Zhang, Z.Y. Han, Y.F. Yuan, L.J. Kang, Novel SA@Ca2+/RCSPs core-shell structure nanofibers by electrospinning for wound dressings, Rsc Advances 8(28) (2018) 15558-15566.

[21] M. Simonet, O.D. Schneider, P. Neuenschwander, W.J. Stark, Ultraporous 3D polymer meshes by low-temperature electrospinning: Use of ice crystals as a removable void template, Polymer Engineering and Science 47(12) (2007) 2020-2026.

[22] B. Sun, Y.Z. Long, F. Yu, M.M. Li, H.D. Zhang, W.J. Li, T.X. Xu, Self-assembly of a three-dimensional fibrous polymer sponge by electrospinning, Nanoscale 4(6) (2012) 2134-2137. 
[23] H. Wang, G.F. Zheng, W.W. Li, X. Wang, D.H. Sun, Direct-writing organic three-dimensional nanofibrous structure, Appl. Phys. A-Mater. Sci. Process. 102(2) (2011) 457-461.

[24] S. Nedjari, G. Schlatter, A. Hébraud, Thick electrospun honeycomb scaffolds with controlled pore size, Materials Letters 142 (2015) 180-183.

[25] G. Yan, J. Yu, Y. Qiu, X. Yi, J. Lu, X. Zhou, X. Bai, Self-Assembly of Electrospun Polymer Nanofibers: A General Phenomenon Generating Honeycomb-Patterned Nanofibrous Structures, Langmuir 27(8) (2011) 4285-4289.

[26] L.D. Wright, R.T. Young, T. Andric, J.W. Freeman, Fabrication and mechanical characterization of 3D electrospun scaffolds for tissue engineering, Biomedical Materials 5(5) (2010) 055006.

[27] C. Zong, Packing, covering and tiling in two-dimensional spaces, Expositiones Mathematicae 32(4) (2014) 297-364.

[28] L. Zhao, J. Yu, W. Li, S. Wang, C. Dai, J. Wu, X. Bai, C. Zhi, Honeycomb porous MnO 2 nanofibers assembled from radially grown nanosheets for aqueous supercapacitors with high working voltage and energy density, Nano Energy 4 (2014) 39-48.

[29] Y. Liu, G. Xu, J. Wei, Q. Wu, X. Li, Cardiomyocyte coculture on layered fibrous sçaffolds assembled from micropatterned electrospun mats, Materials Science and Engineering: C 81 (2017) 500-510.

[30] X. Chen, Y. Xu, M. Liang, Q. Ke, Y. Fang, H. Xu, X. Jin, C. Huang, Honeycomb-like

polysulphone/polyurethane nanofiber filter for the removal of organic/inorganic species from air streams, Journal of Hazardous Materials 347 (2018) 325-333.

[31] S. Thandavamoorthy, N. Gopinath, S.S. Ramkumar, Self-assembled honeycomb polyurethane nanofibers, Journal of Applied Polymer Science 101(5) (2006) 3121-3124.

[32] C.R. Wittmer, A. Hebraud, S. Nedjari, G. Schlatter, Well-organized 3D nanofibrous composite constructs using cooperative, effects between electrospinning and electrospraying, Polymer 55(22) (2014) 5781-5787.

[33] N. Lavielle, A. Hebraud, C. Mendoza-Palomares, A. Ferrand, N. Benkirane-Jessel, G. Schlatter, Structuring and Molding of Electrospun Nanofibers: Effect of Electrical and Topographical Local Properties of Micro-Patterned Collectors, Macromolecular Materials and Engineering 297(10) (2012) 958-968.

[34] T.C. Reis, I.J. Correia, A. Aguiar-Ricardo, Electrodynamic tailoring of self-assembled threedimensional electrospun constructs, Nanoscale 5(16) (2013) 7528-7536.

[35] S. Nedjari, F. Awaja, G. Altankov, Three Dimensional Honeycomb Patterned Fibrinogen Based Nanofibers Induce Substantial Osteogenic Response of Mesenchymal Stem Cells, Scientific Reports 7(1) (2017) 15947.

[36] A. Garcia Garcia, A. Hébraud, J.-L. Duval, C.R. Wittmer, L. Gaut, D. Duprez, C. Egles, F. Bedoui, G. Schlatter, C. Legallais, Poly(E-caprolactone)/Hydroxyapatite 3D Honeycomb Scaffolds for a Cellular Microenvironment Adapted to Maxillofacial Bone Reconstruction, ACS Biomaterials Science \& Engineering 4(9) (2018) 3317-3326.

[37] L.W. Shacklette, C.C. Han, Solubility and Dispersion Characteristics of Polyaniline, MRS Proceedings 328 (1993) 157.

[38] C. Bordes, V. Fréville, E. Ruffin, P. Marote, J.Y. Gauvrit, S. Briançon, P. Lantéri, Determination of poly( $\varepsilon$-caprolactone) solubility parameters: Application to solvent substitution in a microencapsulation process, International Journal of Pharmaceutics 383(1) (2010) 236-243.

[39] J. Stejskal, P. Kratochvíl, N. Radhakrishnan, Polyaniline dispersions 2. UV-Vis absorption spectra, 1993.

[40] A. Haider, S. Haider, I.-K. Kang, A comprehensive review summarizing the effect of electrospinning parameters and potential applications of nanofibers in biomedical and biotechnology, Arabian Journal of Chemistry (2015). 
[41] A.L. Yarin, S. Koombhongse, D.H. Reneker, Taylor cone and jetting from liquid droplets in electrospinning of nanofibers, Journal of applied physics 90(9) (2001) 4836-4846.

[42] L. Persano, A. Camposeo, C. Tekmen, D. Pisignano, Industrial upscaling of electrospinning and applications of polymer nanofibers: a review, Macromolecular Materials and Engineering 298(5) (2013) 504-520.

[43] B. Sun, Y.-Z. Long, F. Yu, M.-M. Li, H.-D. Zhang, W.-J. Li, T.-X. Xu, Self-assembly of a threedimensional fibrous polymer sponge by electrospinning, Nanoscale 4(6) (2012) 2134-2137.

[44] J. STANGER, N. TUCKER, K. KIRWAN, M.P. STAIGER, EFFECT OF CHARGE DENSITY ON THE TAYLOR CONE IN ELECTROSPINNING, International Journal of Modern Physics B 23(06n07) (2009) 1956-1961.

[45] V.F. Achterberg, L. Buscemi, H. Diekmann, J. Smith-Clerc, H. Schwengler, J.-J. Meister, H. Wenck, S. Gallinat, B. Hinz, The Nano-Scale Mechanical Properties of the Extracellular Matrix Regulate Dermal Fibroblast Function, Journal of Investigative Dermatology 134(7) (2014) 1862-1872.

[46] R.L. Mauck, B.M. Baker, N.L. Nerurkar, J.A. Burdick, W.-J. Li, R.S. Tuan, D.M.Elliott, Engineering on the Straight and Narrow: The Mechanics of Nanofibrous Assemblies for Fiber-Reinforced Tissue Regeneration, Tissue Engineering. Part B, Reviews 15(2) (2009) 171-193.

[47] I. Pak, M. Kanthilal, E. Darling, The Role of Cellular Mechanical Properties in MicroenvironmentDependent Behavior, Biophysical Journal 108(2) (2015) 141a.

[48] I.A. Darby, B. Laverdet, F. Bonté, A.J.C. Desmoulière, cosmetic, i. dermatology, Fibroblasts and myofibroblasts in wound healing, 7 (2014) 301.

\section{Graphical Abstract:}

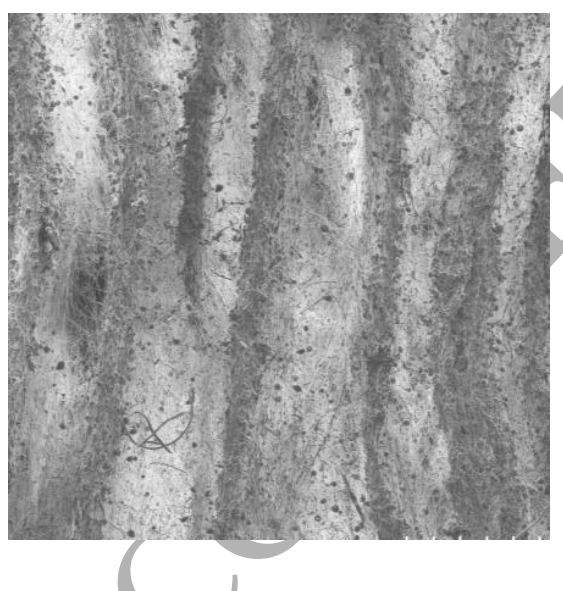

FESEM image of the honeycomb structured nanofibrous scaffold
Seeding scaffolds with Human Dermal Fibroblasts

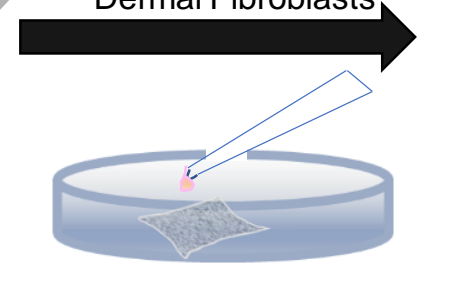

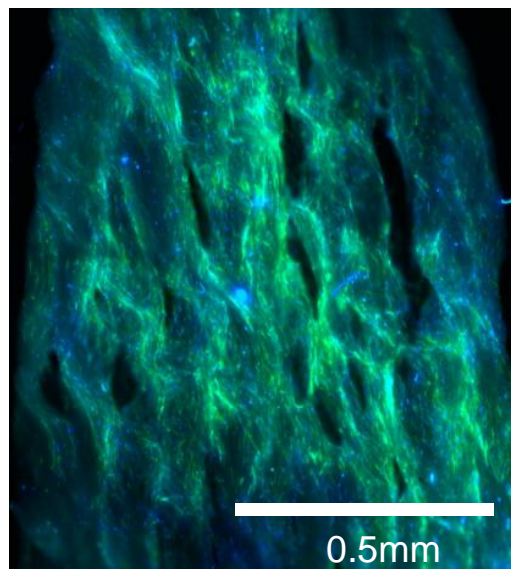

Scaffolds seeded with fibroblasts after Day 7. Blue: nucleus. Green: F-Actin 